\title{
Gold Nanoparticles Enhance the Anticancer Activity of Gallic Acid against Cholangiocarcinoma Cell Lines
}

\author{
Narintorn Rattanata ${ }^{1}$, Sakda Daduang ${ }^{2}$, Molin Wongwattanakul ${ }^{3}$, Chanvit \\ Leelayuwat $^{3,4}$, Temduang Limpaiboon ${ }^{3,4}$, Ratsami Lekphrom ${ }^{5}$, Alisa Sandee ${ }^{6}$, \\ Patcharee Boonsiri ${ }^{7}$, Sirinart Chio-Srichan ${ }^{8}$, Jureerut Daduang ${ }^{3,4 *}$
}

\begin{abstract}
Gold nanoparticles (GNPs) were conjugated with gallic acid (GA) at various concentrations between 30 and $150 \mu \mathrm{M}$ and characterized using transmission electron microscopy (TEM) and UV-Vis spectroscopy (UV-VIS). The anticancer activities of the gallic acid-stabilized gold nanoparticles against well-differentiated (M213) and moderately differentiated (M214) adenocarcinomas were then determined using a neutral red assay. The GA mechanism of action was evaluated using Fourier transform infrared (FTIR) microspectroscopy. Distinctive features of the FTIR spectra between the control and GA-treated cells were confirmed by principal component analysis (PCA). The surface plasmon resonance spectra of the GNPs had a maximum absorption at $520 \mathrm{~nm}$, whereas GNPs-GA shifted the maximum absorption values. In an in vitro study, the complexed GNPs-GA had an increased ability to inhibit the proliferation of cancer cells that was statistically significant $(P<0.0001)$ in both M213 and M214 cells compared to GA alone, indicating that the anticancer activity of GA can be improved by conjugation with GNPs. Moreover, PCA revealed that exposure of the tested cells to GA resulted in significant changes in their cell membrane lipids and fatty acids, which may enhance the efficacy of this anticancer activity regarding apoptosis pathways.
\end{abstract}

Keywords: Gallic acid - gold nanoparticle - anticancer activity - cholangiocarcinoma - nanomedicine

Asian Pac J Cancer Prev, 16 (16), 7143-7147

\section{Introduction}

Cholangiocarcinoma (CCA) is observed in a high percentage in northeast Thailand that are caused by Opistochis viverrini. Surgery by resection is less than $25 \%$ successful in patients (Khan et al., 2002; Thongprasert, 2005). Systemic chemotherapy and radiation therapy have their drawbacks and side effects. There are limits to the standard treatments for CCA, especially when treating patients in the advanced stages. Thus, novel and effective therapeutic agents are urgently needed. Recently, gold nanoparticles (GNPs) have been widely used as chemotherapy delivery vehicles to several cancer cells (Patra et al., 2008; Mohamed et al., 2014; Daduang et al., 2015) due to their sophisticated properties, including their high stability, ultra-small size, facile chemical tailor ability and being biologically non-reactive. Thus, GNPs are applicable as vehicles for the delivery of molecules into cells.

Many studies have also revealed that gallic acid (GA) can inhibit tumor development by several mechanisms including inhibition of metastasis (Ohno et al., 2001); suppression of angiogenesis (Zhao and $\mathrm{Hu}, 2013$ ); induction of apoptosis (You et al., 2010; Chandramohan et al., 2012; Daduang et al., 2015); inhibition of cell viability, proliferation, invasion and tube formation (Lu et al., 2010) and inhibition of migration and invasion (Liao et al., 2012). In this study, we prepared GNPs conjugated with GA. UV-Vis spectroscopy and transmission electron microscopy (TEM) analysis were performed to characterize the GNPs and GNPs-GA. To study the effects of GA on CCA cells (M213 and M214 cell lines), FT-IR microspectroscopy was carried out. The anticancer activities were evaluated using a neutral red assay.

\section{Materials and Methods}

\section{Chemicals and reagents}

Fetal bovine serum, penicillin-streptomycin and trypsin-EDTA were obtained from Gibco BRL (Grand Island, NY). RPMI, gallic acid, neutral red and $\mathrm{HAuCi}^{4}$ were obtained from Sigma-Aldrich Co., LLC (Missouri).

${ }^{I}$ Department of Biochemistry, Faculty of Science, ${ }^{2}$ Faculty of Pharmaceutical Sciences, ${ }^{3}$ Centre for Research and Development of Medical Diagnostic Laboratories, Faculty of Associated Medical Sciences, ${ }^{4}$ Liver Fluke and Cholangiocarcinoma Research Center, Faculty of Medicine, ${ }^{5}$ Department of Chemistry, Faculty of Science, ${ }^{7}$ Department of Biochemistry, Faculty of Medicine, Khon Kaen University, Khon Kaen, ${ }^{6}$ Chulabhorn Research Institute, Bangkok, ${ }^{8}$ Synchrotron Light Research Institute (Public Organization), Nakhon Ratchasima, Thailand*For correspondence: jurpoo@kku.ac.th 
The other chemicals were all of analytical grade.

Synthesis and conjugation of gold nanoparticles (GNPs)

Synthesis of bare GNPs: The gold nanoparticles were fabricated using a method introduced by Turkevich et al. (1951). Ninety five milliliters of $0.01 \% \mathrm{HAuCl}_{4}$ $4 \mathrm{H}_{2} \mathrm{O}$ solution was added to $5 \mathrm{ml}$ of $1 \%$ sodium citrate solution and refluxed for $5 \mathrm{~min}$. The solution was boiled for an additional $30 \mathrm{~min}$ and then cooled down to room temperature. After centrifugation at $12,000 \mathrm{rpm}$ for 10 min, the remaining pellet was dissolved in deionized water to prevent precipitation.

The size and morphology of the GNPs were characterized by TEM using a field emission highresolution transmission electron microscope operating at $200 \mathrm{kV}$ (TECNAI G2 20 Model). GNP samples were prepared for TEM analysis by dropping them onto a carbon film copper grid (Electron Microscopy Sciences, Pennsylvania) and drying in the oven. The absorption spectra at the wavelength range from 400-800 nm were also measured using a spectrometer (Magellan 7 spectrophotometer, TECAN).

Conjugation of GNPs with GA: Two hundred microliters of GNP solution was mixed with $200 \mu \mathrm{m}$ of RPMI completed medium and stirred at $4^{\circ} \mathrm{C}$ overnight. GA was gradually added to the GNPs to a final concentration of 30-150 $\mu \mathrm{m}$. After stirring at $4^{\circ} \mathrm{C}$ overnight, GNPs were centrifuged at $12,000 \mathrm{rpm}$ for $10 \mathrm{~min}$ to remove the unbound GA. The pellet was resuspended in RPMI completed medium $(200 \mu \mathrm{m})$ and immediately used for biological assays.

Gold nano-conjugation evaluation: $i$ ) Measurement of GNPs-GA size by spectrophotometry. Surface plasmon resonance band measurements using UV-Vis spectroscopy (Magellan 7 spectrophotometer, TECAN) were used to detect the change in size using bare GNPs to GNPs-GA. The optical absorption spectra were observed in the wavelength range of 400-800 nm. ii) Measurement of the concentration of unbound GA after GNPs-GA conjugation by high performance liquid chromatography (HPLC).

Evaluation of GNPs-GA conjugation efficiency: The percentage of GNPs-GA conjugation was indirectly determined by measuring the unbound GA. Twenty microliters of the supernatant from the GNPs-GA conjugation step was injected into an HPLC C18 Luna column (15 cm x $3.0 \mathrm{~mm}$, Phenomenex, California). Gradient elution was conducted at a flow rate of $1.5 \mathrm{ml} /$ min using methanol and $0.5 \%$ phosphoric acid at ratios of 5:95, 70:30, 90:10 and 5:95 at times 0-17, 17-18, 18-20.5 and $20.5-25 \mathrm{~min}$, respectively. The absorbance at $270 \mathrm{~nm}$ was detected by a spectrometer (UV 2479, Waters), and the peaks were analyzed by the Clarity program (Waters). One hundred and fifty micromolar GA was used as a standard compound. The mean peak areas from the unbound GA in the supernatant were calculated by comparison with the standard GA peak area.

\section{Cell culture}

Human cholangiocarcinoma (CCA) cell lines, namely M213 and M214, were obtained from Prof. Banchob Sripa, Department of Pathology, Faculty of Medicine,
Khon Kaen University. They were cultured in RPMI 1640 medium supplemented with $10 \%$ FBS and $1 \%$ penicillin-streptomycin (Sigma-Aldrich) at $37^{\circ} \mathrm{C}$ in a $\mathrm{CO}_{2}$ incubator (95\% relative humidity, $5 \% \mathrm{CO}_{2}$ ). Cell lines were trypsinized with $1 \mathrm{X}$ trypsin-EDTA when they reached confluence.

\section{Cell viability and $\mathrm{IC}_{50}$ determination}

A neutral red (NR) assay modified from Borenfreund and Puerner (1984) was used to testfor cell viability. Briefly, cell lines were moved from a culture flask using $1 \mathrm{X}$ trypsin-EDTA. Then, RPMI supplemented with 10\% FBS was added. The cells were counted using a hemocytometer and approximately 6,000 cells were seeded per well in a 96-well plate. The cells were cultured in completed RPMI 1640 medium at $37^{\circ} \mathrm{C}$ in a $\mathrm{CO}_{2}$ incubator for $24 \mathrm{hr}$. All of the cell lines were treated with varying concentrations (30, $50,70,90,110,120$ and $150 \mu \mathrm{M})$ of GA and GNPs-GA and incubated for an additional $24 \mathrm{hr}$. NR was added to the tested cells for $3 \mathrm{hr}$. Cell viability was detected at 540 $\mathrm{nm}$ using an RT-2100c micro plate reader (Rayto, China).

\section{Studying the effect of GA on cell lines by FTIR analysis}

Studying the effect of GA on cell lines by Fourier transform infrared spectroscopy (FTIR) analysis

Cancer cell coated IR Low-e slides (Kevley Technologies, Chesterland, $\mathrm{OH}$ ) were prepared by adding Low-e slides to each well in a cell culture plate. Then, cell line M214 was cultured according to the above method. GA was added at the $\mathrm{IC}_{50}$ concentration. After $12 \mathrm{hr}$, the slides were collected and gently washed twice with $0.85 \%$ sodium chloride. The slides were air-dried and kept in a desiccator prior to FTIR microscopic analysis (Bruker Optik GmbH, Ettlingen, Germany). The cancer cell coated Low-e slides were placed under the microscope objective and IR spectra were recorded in reflection mode from 4000 to $400 \mathrm{~cm}^{-1}$ at a spectral resolution of $4 \mathrm{~cm}^{-1}$. Spectra from all of the samples were extracted using the macro converter in the OPUS5.5 software Bruker Optik GmbH, Ettlingen, Germany) prior to multivariate data analysis using the Unscrambler® 9.7 software (Camo Inc., Oslo, Norway). Approximately 50 representative spectra from each sample were pretreated by performing the second derivative, which was carried out by applying the Savitzky-Golay algorithm with 13 smoothed data points and normalized with Extended Multiplicative Signal Correction (EMSC). Pretreated spectra were then analyzed by principle component analysis (PCA) to distinguish between the control and GA-treated cells.

\section{Results}

\section{Determination of gold nanoparticle size}

The obtained bare GNPs were ruby red, indicating that nanoparticles formed. The average diameter of these GNPs was approximately $16-20 \mathrm{~nm}$, which was evaluated using the Image $\mathrm{J}$ software $1.48 \mathrm{~V}$ by randomly selecting 100 GNPs from a TEM photograph as shown in Figure 1 .

Conjugation of gold nanoparticles with gallic acid GNP-GA formation was evaluated using a UV-Vis 
spectrophotometer. As shown in Figure 2, GNPs have a maximum absorption at $524 \mathrm{~nm}$, whereas the maximum absorption value of GNPs-GA has shifted, confirming that the GNPs have conjugated with GA; the conjugation also caused plasmon resonance spectrum shifting. However, TEM results did not show a significant increase in the particle size for GNPs-GA compared to that for the bare GNPs (data not shown).

Measurement of the concentration of unbound gallic acid after GNPs-GA conjugation by HPLC

To quantitate the level of GNPs-GA conjugation via an indirect method, the supernatant that was discarded after the GNPs-GA conjugation step was tested for unbound GA by HPLC. Compared to the GA standard retention time at $7.49 \mathrm{~min}$, the supernatant had no peak at this retention time (Figure 3). These results show that GNPs-GA conjugation was completed.

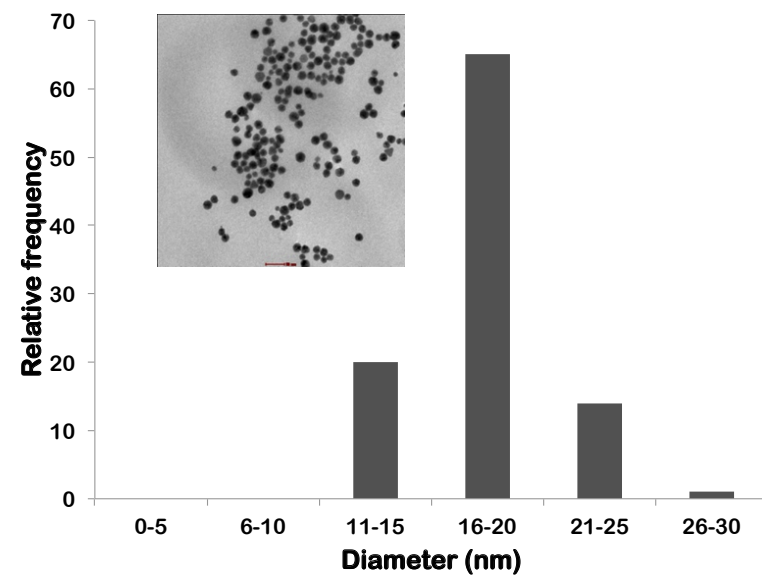

Figure 1. Transmission Electron Microscopy Image of GNPs at a Magnification of 100,000X. The scale bar denotes $50 \mathrm{~nm}$. The average diameter of the GNPs is approximately $16-20 \mathrm{~nm}$

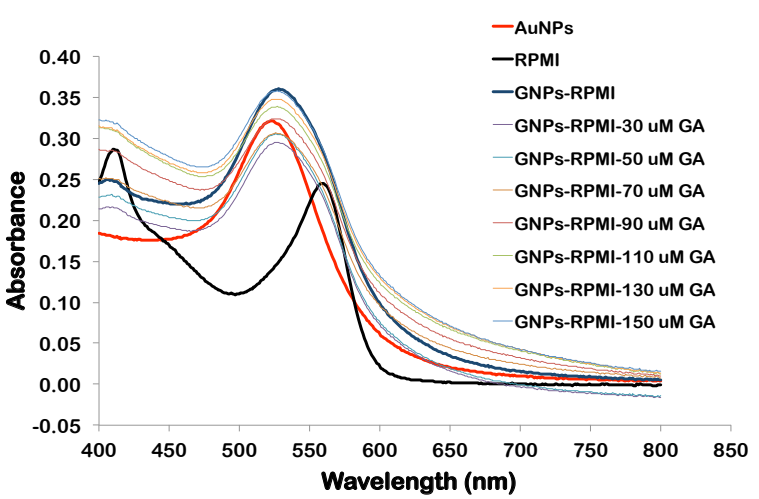

Figure 2. Surface Plasma Resonance Spectra of GNPs before and after Surface Conjugation with Gallic Acid (GNPs-GA) using a UV-visible Spectrophotometer. Scanning occurred in the range from 400-800 nm
The anticancer activity of GNPS-GA and GA on cholangiocarcinoma cell lines

The cytotoxicity test was performed using a neutral

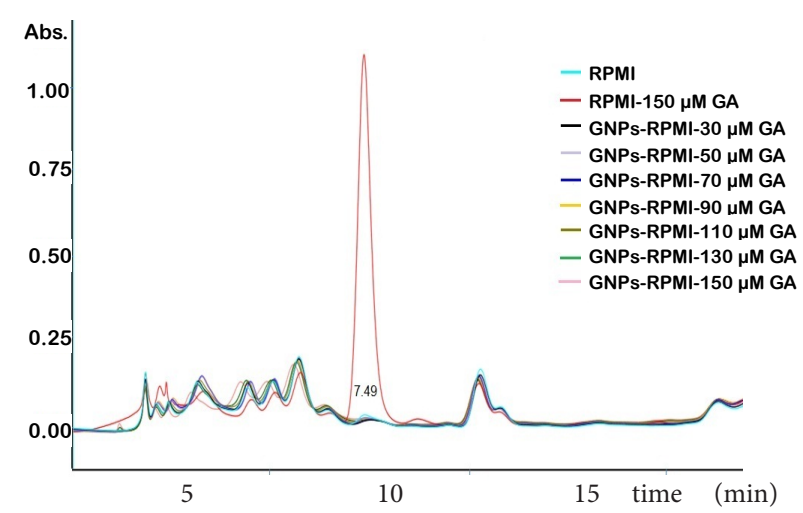

Figure 3. HPLC Chromatogram of Gallic Acid Standard (red line), the Supernatant that was Discarded after the GNP-GA Conjugation step (Other lines) and the Negative Control RPMI (green line)
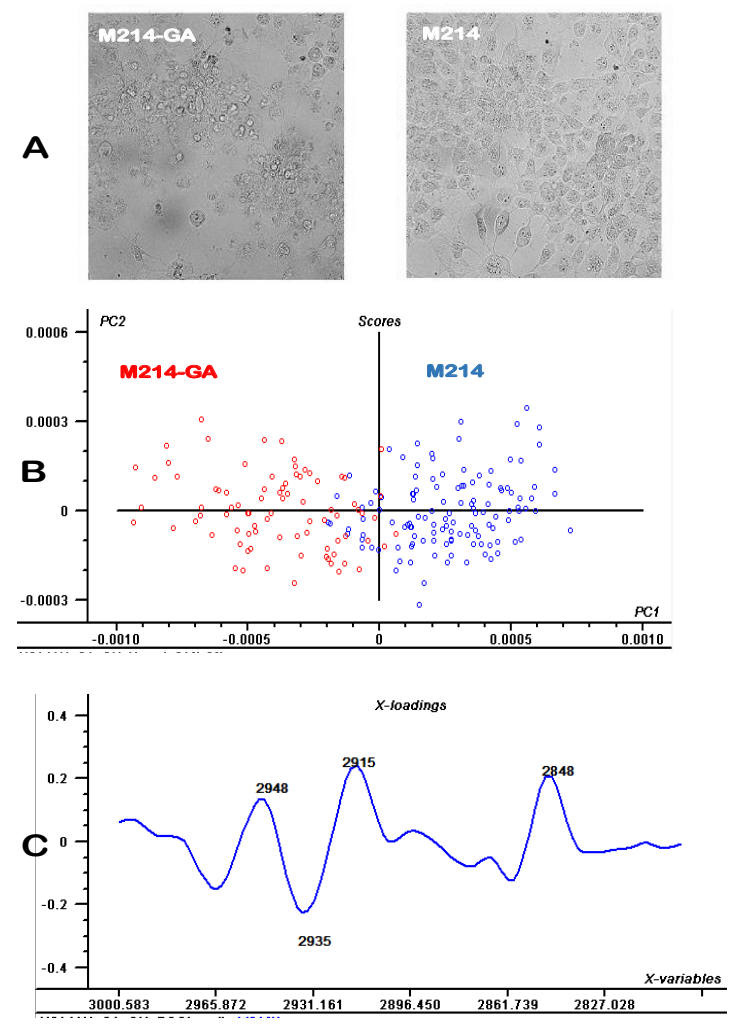

Figure 4. FTIR Spectra Analysis of the wave Region from 3000 to $2800 \mathrm{~cm}^{-1}$ using Principle Component Analysis (PCA) from the Control and GA-tested Cells in M214 Cells. (A) Morphological changes in M214 cells induced by GA at $150 \mu \mathrm{M}$ for $12 \mathrm{hr}$ with capture by a phasemicroscope with a $100 \mathrm{X}$ objective. (B) PCA score plot. (C) Loading plots

Table 1. The IC $_{50}$ Values for GA and GNPs-GA on CCA Cell Lines

\begin{tabular}{|c|c|c|c|c|}
\hline \multirow[t]{2}{*}{ Cell lines } & \multirow[t]{2}{*}{ Histological types } & \multicolumn{3}{|c|}{$\mathrm{IC}_{50}(\mu \mathrm{M})$} \\
\hline & & GA & GNPs-GA & $\%$ Change \\
\hline M213 & Well-differentiated adenocarcinoma & 119 & 94 & 21 \\
\hline M214 & Moderately differentiated adenocarcinoma & 147 & 111 & 25 \\
\hline
\end{tabular}


red assay. Surprisingly, the cells treated with GNPs-GA, GA had decreased viability compared to cells treated with GA alone in both M213 and M214 cells. The $\mathrm{IC}_{50}$ concentration for the GNPs-GA is lower than that of GA, with the difference being about $21 \%$ and $25 \%$ for M213 and M214 cells, respectively (Table 1).

The effect of GA on CCA cell lines by Fourier transform infrared spectroscopy (FTIR) analysis

Phase-contrast micrographs demonstrated the morphologic changes between the control and GA-treated cells (Figure 4A). An FTIR microspectroscopy technique was applied to study the GA mechanism of action because of its sensitivity, rapidity, low expense cost and simplicity. PCA score plots showed distinct group separation along PC1 when comparing the spectra from M214 (control cells; blue dots) and M214-GA (tested with GA; red dots), as shown in Figure 4B. In the loading plots corresponding to this comparison, $\mathrm{PC} 1$ and $\mathrm{PC} 2$ separately controlled and tested the spectra for changes in the lipid signal (2948, 2835, 2915, and $2848 \mathrm{~cm}-1$ ), as shown in Figure 4C. Spectral data from the PCA revealed segregation between the control and GA-treated cells.

\section{Discussion}

In the present study, the effects of GA were investigated in human CCA cell lines (M213, and M214). Differences in response to two agents were observed in 2 cell lines. M213 cells are well-differentiated adenocarcinoma cells with higher susceptibility to both GA and GNPs-GA than M214 cells (moderately differentiated adenocarcinoma cells). Tepsiri et al., 2005 revealed that sensitivity to chemotherapeutic agents is not associated with CCA histological type. Daduang et al., 2015 found that GA induced apoptosis via the intrinsic pathway and the extrinsic pathway II. The extrinsic pathways II activates mitochondrial outer membrane permeabilization (MOMP) by relying on the apoptogenic factors cytochrome $c$ and Smac/DIABLO, which are released from the mitochondria, leading to the activation of effector caspases and cell death (Schug et al., 2011). The mechanism of apoptosis undertaken by the cells treated with GA was determined using FTIR microspectroscopy. A good marker for apoptosis is the lipid signal $(2948,2835,2915$, and $2848 \mathrm{~cm}^{-1}$ ), which is exposed to the outer leaflet of the plasma membrane by phosphatidylserine. The results from the FTIR microspectroscopy agreed with a previous report by Gaudenzi et al., (2004). Thus, GA is proposed to initiate MOMP, which progressively leads to apoptosis.

TEM images of the GNPs and GNPs-GA were taken (data not shown), though they do not show significant differences in the sizes of the particles. However, the GNP and GA could form a core-shell structure with a thin GA shell that may not be apparent in the TEM image due to the low contrast between GA and the sample substrate. A similar case has been reported for a TEM image of Au-polymer core shell particles (Mandal et al., 2002). The shifts in the plasmon resonance spectrum of GNPs and GNPs-GA (Figure 2) and the HPLC results (Figure3) confirm the success of the conjugation. The results agree with a previous study by Moreno-Alvarez et al., 2010, which demonstrated that the maximum absorption of GA-stabilized GNPs shifted to a broader band. In this study, a cytotoxicity test was used to examine M213 and M214 cells treated with GA and GNPs-GA. The $\mathrm{IC}_{50}$ for the GNPs-GA was lower than that of GA alone (Table1). This result is in agreement with Daduang et al., 2015, who reported that gold nanoparticles have the potential to be used as phytochemical delivery agents for alternative cancer treatments.

GNPs conjugated with GA performed well in RPMI medium because the serum in the medium helps transport the GNPs-GA into cell lines, as described by Chithrani et al., 2006.

In conclusion, gold nanoparticles have the potential to be used as phytochemical delivery agents for alternative cancer treatments. To enhance the anticancer activity of gold nanoparticles in CCA lines, GNP surfaces can be functionalized with gallic acid. We also provide data that may suggest that FTIR microscopy can distinguish CCA cells that have undergone apoptosis.

\section{Acknowledgements}

This work was supported by the Higher Education Research Promotion and National Research University Project of Thailand, Office of the Higher Education Commission through the Center of Excellence in Specific Health Problems in the Greater Mekong Subregion cluster (SHeP-GMS), Khon Kaen University, Thailand (Ph.D.54208 to Rattanata N and NRU542014 to Daduang J). The authors thank the Liver Fluke and Cholangiocarcinoma Research Center for providing the CCA cell lines.

\section{References}

Borenfreund E and Puerner JA (1985). A simple quantitative procedure using monolayer cultures for cytotoxicity assays (HTD/NR-90). J Tissue Cult Met, 9, 7-9.

Chandramohan RT, Bharat RD, Aparna A, et al (2012). Antileukemic effects of gallic acid on human leukemia K562 cells: downregulation of COX-2, inhibition of BCR/ABL kinase and NF- $x$ B inactivation. Toxicol In Vitro, 26, 396-405.

Chithrani BD, Ghazani AA and Chan WC (2006). Determining the size and shape dependence of gold nanoparticle uptake into mammalian cells. Nano Lett, 6, 662-8.

Daduang J, Palasap A, Daduang S, et al (2015). Gallic acid enhancement of gold nanoparticle anticancer activity in cervical cancer cells. Asian Pac J Cancer Prev, 16, 169-74.

Gaudenzi S, Pozzi D, Toroa P, et al (2004). Cell apoptosis specific marker found by fourier transform infrared spectroscopy. Spectroscopy, 18, 415-22.

Khan S, Davidson BR, Goldin R, et al (2002). Guidelines for the diagnosis and treatment of cholangiocarcinoma: consensus document. Gut, 51, 1-9.

Liao CL, Lai KC, Huang AC, et al. (2012). Gallic acid inhibits migration and invasion in human osteosarcoma U-2 OS cells through suppressing the matrix metalloproteinase-2/-9, protein kinase $\mathrm{B}(\mathrm{PKB})$ and $\mathrm{PKC}$ signaling pathways. Food Chem Toxicol, 50, 1734-40.

Lu Y, Jiang F, Jiang H, et al (2010). Gallic acid suppresses cell viability, proliferation, invasion and angiogenesis in human glioma cells. Eur J Pharmacol, 641, 102-7. 
Mandal TK, Fleming MS and Walt DR (2002). Preparation of polymer coated gold nanoparticles by surface confined living radical polymerization at ambient temperature. Nano Lett, 2, 3-7.

Mohamed MS, Veeranarayanan S, Poulose AC, et al (2014). Type 1 ribotoxin-curcin conjugated biogenic gold nanoparticles for a multimodal therapeutic approach towards brain cancer. Biochim Biophys Acta, 1840, 1657-69.

Moreno-Alvarez SA, Martinez-Castanon GA, Nino-Martinez $\mathrm{N}$; et al (2010). Preparation and bactericide activity of gallic acid stabilized gold nanoparticles. J Nanopart Res, 12, 2741-6.

Ohno T, Inoue M and Ogihara Y (2001). Cytotoxic activity of gallic acid against liver metastasis of mastocytoma cells P-815. Anticancer Res, 21, 3875-80.

Patra CR, Bhattacharya R, Wang E, et al (2008). Targeted delivery of gemcitabine to pancreatic adenocarcinoma using cetuximab as a targeting agent. Cancer Res, 68, 1970-8.

Schug ZT, Gonzalvez F, Houtkooper RH,Vaz FM, Gottlieb E (2011). BID is cleaved by caspase- 8 within a native complex on the mitochondrial membrane. Cell Death Differ, 18, 538-48.

Tepsiri N, Chaturat L, Sripa B, et al (2005). Drug sensitivity and drug resistance profiles of human intrahepatic cholangiocarcinoma cell lines. World J Gastroenterol, 11, 2748-53.

Thongprasert S (2005). The role of chemotherapy in cholangiocarcinoma. Ann oncol, 16, 93-6.

Turkevich J, Stevenson PC and Hillier J (1951). A study of the nucleation and growth processes in the synthesis of colloidal gold. Discuss. Faraday Soc, 11, 55-75.

You BR, Moon HJ, Han YH, Park WH (2010). Gallic acid inhibits the growth of HeLa cervical cancer cells via apoptosis and/or necrosis. Food Chem Toxicol, 48, 1334-40.

Zhao B and Hu M (2013). Gallic acid reduces cell viability, proliferation, invasion and angiogenesis in human cervical cancer cells. Oncol Lett, 6, 1749-55. 